University of Nebraska - Lincoln

DigitalCommons@University of Nebraska - Lincoln

USDA National Wildlife Research Center - Staff

Publications

U.S. Department of Agriculture: Animal and Plant Health Inspection Service

2013

\title{
The Cost of Canine Rabies on Four Continents
}

A. M. Anderson

USDA/APHIS/WS National Wildlife Research Center, aaron.m.anderson@aphis.usda.gov

S. A. Shwiff

USDA/APHIS/WS National Wildlife Research Center, stephanie.a.shwiff@aphis.usda.gov

Follow this and additional works at: https://digitalcommons.unl.edu/icwdm_usdanwrc

Anderson, A. M. and Shwiff, S. A., "The Cost of Canine Rabies on Four Continents" (2013). USDA National Wildlife Research Center - Staff Publications. 1236.

https://digitalcommons.unl.edu/icwdm_usdanwrc/1236

This Article is brought to you for free and open access by the U.S. Department of Agriculture: Animal and Plant Health Inspection Service at DigitalCommons@University of Nebraska - Lincoln. It has been accepted for inclusion in USDA National Wildlife Research Center - Staff Publications by an authorized administrator of DigitalCommons@University of Nebraska - Lincoln. 
ORIGINAL ARTICLE

\title{
The Cost of Canine Rabies on Four Continents
}

\author{
A. Anderson and S. A. Shwiff \\ USDAVAPHIS/WS National Wildlife Research Center, Fort Collins, CO, USA
}

\author{
Keywords: \\ canine rabies; cost; economics; Latin America; \\ Africa; Asia

\section{Correspondence:} \\ A. Anderson. USDA/APHIS/WS National \\ Wildlife Research Center, 4101 LaPorte Ave., \\ Fort Collins, CO 80521, USA. \\ Tel.: + 1(970)266-6264; \\ Fax: + 1(970)266-6089; \\ E-mail: Aaron.M.Anderson@aphis.usda.gov ph
}

Received for publication July 15, 2013

doi:10.1111/tbed.12168

\begin{abstract}
Summary
We estimated the economic impacts of canine rabies in Latin America, Africa and Asia. Direct and indirect costs of rabies post-exposure prophylaxis, dog vaccination and control, rabies diagnostic testing and cattle mortality-related costs were accounted for. The number of human deaths was updated from previous estimates based on population growth, and the costs associated with the risk of human mortality were incorporated. We accounted for uncertainty associated with the parameter estimates using a Monte Carlo simulation and estimated that the global burden of canine rabies is approximately $\$ 124$ billion annually. This result illustrates the potential benefits that could be realized if canine rabies was eliminated and provides an important benchmark against which the cost of any potential elimination campaign can be compared.
\end{abstract}

\section{Introduction}

The World Health Organization estimates that, on average, about 55000 people die of rabies each year, and $99 \%$ of these deaths are attributable to canine rabies in Asia and Africa. There are significant differences in the status of the disease in different parts of the world. In the United States, extensive dog vaccination and public education efforts began after World War II, and the disease was eliminated in the 1970s (Blanton et al., 2010). Much of Western and Central Europe is also free from the disease, although it remains a threat in Eastern Europe and around the periphery (Bourhy et al., 2005). Many countries in Latin America have made considerable progress in recent decades, and the disease is now prevalent only in Haiti and Bolivia (Schneider et al., 2007; Global Alliance for Rabies Control, 2012). These examples of progress and elimination contrast with the situation in much of Africa and Asia where the threat posed by the disease has increased in recent years (Lembo et al., 2010).

The progress in Latin America is largely a result of mass vaccination of dogs, but in parts of Africa and Asia, there remain obstacles to successful implementation of this strategy. These obstacles may include the prevalence of inaccessible dogs, the inability of owners to bring dogs in for vaccination, lack of information about dog populations, lack of canine rabies surveillance and diagnostic capabilities and insufficient resources from veterinary services (Lembo et al., 2010). Yet the potential benefits of limiting or eliminating the disease are large; in Asia alone, Knobel et al. (2005) estimates that 7.5 million people receive rabies postexposure prophylaxis (PEP) each year as a result of potential exposure to canine rabies. The cost associated with PEP could be avoided if the disease was eradicated. Additional costs imposed by the disease include the cost of testing dogs and other animals potentially exposed, the ongoing cost of sporadic dog vaccination and the income losses due to livestock mortality.

There are two aspects of the economics of canine rabies that have received little attention. The first is the cost of the mortality risk associated with the presence of the disease. While the cost of mortality risk cannot be directly observed, the implicit value of a reduction in mortality risk has been studied extensively by observing choices people make that reflect how they value reductions in risk. These choices may involve employment decisions (e.g. employment that involves relatively high mortality risk but relatively high pay) or decisions to purchase or use certain consumer goods that affect mortality risk (e.g. purchase of a relatively safe car that is also relatively more expensive). Ultimately, a cost of risk can be derived by estimating how much people are willing to pay for a reduction in that risk. 
The purpose of this study was to extend current estimates of the economic impact of canine rabies by accounting for remaining impacts in Latin America and incorporating the cost of human mortality risk. These additions provide a more complete assessment of the current global impacts of the disease and also provide a better estimate of the potential benefits that could be realized if the prevalence of the disease is reduced or it is eliminated. Shwiff et al. (2013) provided a qualitatively focused account of many of the impacts of canine rabies; the present analysis complements Shwiff et al. (2013) by providing a more detailed and quantitative account of the current impacts in Africa, Asia and Latin America. From a One Health perspective, this analysis is important because it considers that impacts of the disease are linked to companion animals, livestock and humans. It also provides information that can be used to better educate stakeholders and the public about the true cost of the disease which is an important component of the One Health approach to management efforts.

\section{Materials and Methods}

\section{Direct impacts}

Estimation of the global economic impact of canine rabies required coalescing information from several sources on the direct and indirect impacts of disease in different regions of the world. Specifically, estimates of human mortality, PEP, animal testing, dog vaccinations and livestock mortality were required, along with the cost associated with each. Here, we relied on estimates provided for Africa and Asia by Knobel et al. (2005). However, population estimates were updated, and assumptions about PEP use were altered to reflect the continued movement away from the use of nerve tissue vaccine.

Annual human mortality estimates in Africa and Asia were extrapolated from Knobel et al. (2005) by scaling based on population growth. Mortality estimates in the study by Knobel et al. considered the human populations at risk and dog population densities. Because the human populations at risk and dog population densities are both a function of the total human population, we scaled the original estimates by the population growth that has occurred since the original study. The updated human mortality estimates for Africa and Asia are 31329 and 38 090, respectively (Table 1). Estimation of human mortality from canine rabies in Latin America was based on information from the Global Alliance for Rabies Control, which reports that the majority of remaining cases occur in Haiti and Bolivia. From 2008 to 2011, a total of 59 people died of rabies in Latin America, yielding an average annual mortality of about 15 (Global Alliance for Rabies Control, 2012).

Estimates of annual PEP use in Africa and Asia from Knobel et al. (2005) were scaled by population growth yielding estimates of 264325 PEP cases in Africa and 8709365 in Asia. The number of annual PEP cases due to potential exposure of canine rabies in Latin America was estimated based on data from Schneider et al. (2007). While the prevalence of canine rabies in Latin America has fallen dramatically in recent decades, the perceived risk remains, and it is likely that PEP use remains elevated. During 1990-2003, an average of 961195 people were reported to receive PEP in Latin America. During the same time period, dogs accounted for $65 \%$ of actual rabies cases with information on the attacking animal (Schneider et al., 2007). Multiplying the average number of annual PEP cases by 0.65 yielded our estimate of 624777 annual PEP cases attributable to dogs in Latin America.

Our estimates of the number of rabies diagnostic tests performed each year in Africa and Asia are 5300 and 16500 , respectively, which are sourced without adjustment from Knobel et al. (2005) because it is not clear that diagnostic testing necessarily varies with population size. Our estimate for the annual number of tests in Latin America was 73 752, which was the average number of canine samples tested for rabies during the time period 1990-2003 (Schneider et al., 2007). Estimates of the number of dogs vaccinated annually came from Knobel et al. (2005) and Schneider et al. (2007), which reported 6.7 million, 40 million and 42 million dogs vaccinated annually in Africa, Asia and Latin America, respectively.

Knobel et al. (2005) estimated annual cattle mortality of 11500 and 21150 in Africa and Asia, respectively. We used these without adjustment because the relationship between cattle loss and human population is unknown. Data on cattle loss in Latin America were unavailable. We therefore multiplied cattle population estimates from Haiti and Bolivia by an estimated mortality rate that was constructed by adjusting the cattle mortality rate for Africa and Asia based on the ratio of the human mortality rate in Haiti and Bolivia to the human morality rate in Africa and Asia combined. Specifically, we estimated a cattle mortality rate of $2.7 \times 10^{-6}$ which implies annual cattle loss of about 24 head in Haiti and Bolivia due to canine rabies.

\section{Unit costs}

Costs associated with PEP were based on estimates from Knobel et al. (2005), although several adjustments were made. There has been continued movement away from nerve tissue vaccines in many countries. Notably, India discontinued production of nerve tissue vaccine in 2005, and a number of other Asian countries also discontinued production of nerve tissue vaccine during the 1990s and 2000s (Tenzin and Ward, 2012). We therefore assumed all patients receive tissue culture vaccine on the grounds that use of nerve tissue vaccine is minimal and decreasing. 
Adjusting for inflation, the cost of intramuscular vaccination was estimated to be $\$ 11.90$ per dose, and the cost of intradermal vaccine is estimated to be $\$ 2.98$ per dose (Knobel et al., 2005). We also assumed that patients who are vaccinated intramuscularly will receive three doses over three visits, on average, and those who receive the intradermal vaccine will receive six doses over three visits (Knobel et al., 2005). Note that although these are fewer visits and doses than required by current WHO guidelines, it is expected that some patients will not be willing or able to follow those guidelines. Material costs per injection are estimated at $\$ 0.12$, and overhead costs per visit are estimated at $\$ 0.60$ (Knobel et al., 2005). We assumed the same costs for all regions.

Sufficient data do not exist to precisely estimate the percentage of patients who receive intradermal vaccination. Knobel et al. (2005) assumed that $0 \%$ of African patients and only $3 \%$ of Asian patients received intradermal vaccine. However, there has been a movement towards the intradermal vaccine in some countries in recent years due to its cost advantage (Sudarshan et al., 2006). We arbitrarily assumed that $3 \%$ of African patients and $6 \%$ of Asian and Latin American patients receive intradermal vaccination.

A small percentage of patients receive rabies immunoglobulin (RIG). Adjusted for inflation, the cost of human rabies immunoglobulin (hRIG) was assumed to be $\$ 130.98$ per dose, and the cost of equine rabies immunoglobulin (eRIG) was assumed to be $\$ 29.77$ per dose (Knobel et al., 2005). Consistent with Knobel et al. (2005), we assumed $1 \%$ and $6 \%$ of patients receive RIG in Africa and Asia, respectively. In Latin America, we assumed 3.5\% based on the average of the estimates for Africa and Asia. Additionally, $0 \%$ of African patients receive hRIG, while $10 \%$ of Asian patients who receive RIG receive hRIG (Knobel et al., 2005). We again relied on the average and assumed 5\% of Latin American patients who receive RIG receive hRIG. The resulting expected PEP cost per case ranged from $\$ 37.64$ in Africa to $\$ 39.21$ in Asia (Table 2). The expected PEP costs were calculated as the probabilityweighted summation of the various costs and account for all injections and visits.

Patients also incur indirect costs associated with transportation and lost wages as a result of PEP treatment (Shwiff et al., 2007). We assumed each patient and person accompanying them loses 0.5 days of work, but we assumed only $20 \%$ of patients are accompanied by an adult to provide a more conservative estimate than Knobel et al. (2005) (Table 3). We also assumed 20\% of visitors will not suffer any income loss because they are either unemployed or employed in an occupation that enables them to avoid the opportunity cost of missed work. Patients also incur transport costs, which we assumed to be $\$ 2.38$ and $\$ 4.52$ per visit for Africa and Asia, respectively (Knobel et al.,
2005). Transport costs in Latin America were set at the average of those in Africa and Asia.

Estimates of dog vaccination costs, dog control costs, rabies diagnostic costs and cattle value were sourced primarily from Knobel et al. (2005) (Table 4). Vaccination and testing costs in Latin America were assumed equal to those reported for Africa and Asia, and the value of cattle was assumed to be the average of the values reported for Africa and Asia. No estimates of dog control costs were available for Latin America.

\section{Value of a statistical life}

The costs described above are easily understood as costs because they represent monetary expenses. Additionally, they are easily estimated based on observed market prices or production costs. The human mortality risk associated with canine rabies also represents a cost, albeit one that is not readily observed. It is clear that people value a reduction in mortality risk. People pay more for relatively safe cars, organic food and many other products that they perceive to reduce their own mortality risk; they also spend money on rabies PEP. Yet people engage in many risky behaviours such as smoking, driving without a seatbelt or working in a dangerous occupation that indicate that the value they place on reduced mortality risk is not infinite.

Statistical analyses of price and wage data allow the determination of how prices and wages are affected by mortality risk. This reveals how much people are willing to pay for reduced mortality risk or, conversely, how much people must be compensated to tolerate increased risk. That is, it reveals the value people place on a change in mortality risk. At the social level, a change in mortality risk will translate into more or fewer deaths. It is therefore possible to place a cost or benefit on a change in the number of human deaths by valuing the underlying change in mortality risk. The resulting estimate of the cost of human death and the underlying change in mortality risk that causes it is termed the value of a statistical life (VSL). Note that the VSL does not attempt to value a particular individual's life and is not based on income or earnings potential. Instead, it reflects a society's acceptance of mortality risk.

There is considerable variation in VSL estimates, but a number of extensive meta-analyses have been performed. Adjusting estimates to 2011 US dollars, Miller (2000) estimated VSL at \$5.1 million, Viscusi and Aldy (2003) estimated it at \$9.1 million and Mrozek and Taylor (2002) provided a value of $\$ 2.7$ million. Additionally, the average VSL estimate from 37 studies included in Bellavance et al. (2009) was \$12.4 million. However, these estimates are likely overstating the true value (Doucouliagos et al., 2012). By correcting for publication selectivity bias, Doucouliagos et al. (2012) arrive at an estimate of \$2.2 million. 
Consideration of the effects of including workers' compensation, time trends and workers' average income yields an even lower estimate of $\$ 1.8$ million (Doucouliagos et al., 2012). We assumed a VSL of $\$ 1.8$ million because this was the most recent and thorough estimate available from a meta-analysis, and VSL estimates from developing countries are often lower than those from developed countries (Bowland and Beghin, 2001; Hoffman et al., 2012).

\section{Monte Carlo simulation}

The final calculation of the total cost of canine rabies was based on a 39 parameters (for the three regions: human deaths, VSL, PEP cases, expected direct PEP cost per case, expected income loss per PEP case, expected travel costs per PEP case, dog vaccine quantity and cost, diagnostic test quantity and cost, dog control costs, cattle deaths and cattle value). Because there is uncertainty about the true value of these parameters, a Monte Carlo simulation was performed to calculate the total cost and illustrate how uncertainty affects the results. Although we could not characterize the degree of uncertainty in any of the parameter estimates, we made an arbitrary assumption that each is distributed triangularly with the mean equal to our point estimate and minimum and maximum values set at $\pm 25 \%$ of the point estimate. Note that this distributional assumption need not reflect the distribution of a certain parameter as it is observed across regions or time; it is simply meant to capture some of the uncertainty associated with each parameter and illustrate how different parameter estimates affect the results.

The simulation proceeded by randomly drawing values based on the assumed distribution and the parameter values of that distributions. The simulations were performed in Microsoft Excel, and the rand() function was used to generate random numbers. Random draws from a triangular distribution were simulated by generating a random number on the uniform interval $[0,1]$ and evaluating the inverse of the triangular cumulative distribution function (Equation 1) at that number.

$F^{-1}(y)=\left\{\begin{array}{cl}L+\sqrt{y(M-L)(U-L)} & \text { for } 0<y<(M-L) /(U-L) \\ U-\sqrt{(1-y)(U-M)(U-L)} & \text { for }(M-L) /(U-L)<y<1\end{array}\right.$

Given the drawn values of the 39 variables, the total cost was calculated. This process was repeated 100000 times to sufficiently characterize the mean, median and variance of the results.

\section{Results}

Given the distributional assumptions made in the Monte Carlo simulation, the expected total annual cost of canine rabies is approximately $\$ 124.2$ billion ( $\mathrm{SD}=12$ billion), and the median cost was $\$ 123.8$ billion (Fig. 1). Human mortality risk accounts for the vast majority of the total cost ( $\$ 123.4$ billion or $99.3 \%$ of all costs). Human mortality in Asia accounts for about 55\% of the total human mortality costs, and Africa accounts for nearly all of the remainder. Excluding the cost of human mortality risk, monetary expenses associated with canine rabies total $\$ 832$ million, of which about $82 \%$ occur in Asia (Table 5).

\section{Discussion}

Our analysis indicates that the global cost of canine rabies is approximately $\$ 124.2$ billion annually. In order of contribution, this total is attributable to human mortality risk, direct PEP costs, lost income due to PEP, dog vaccination

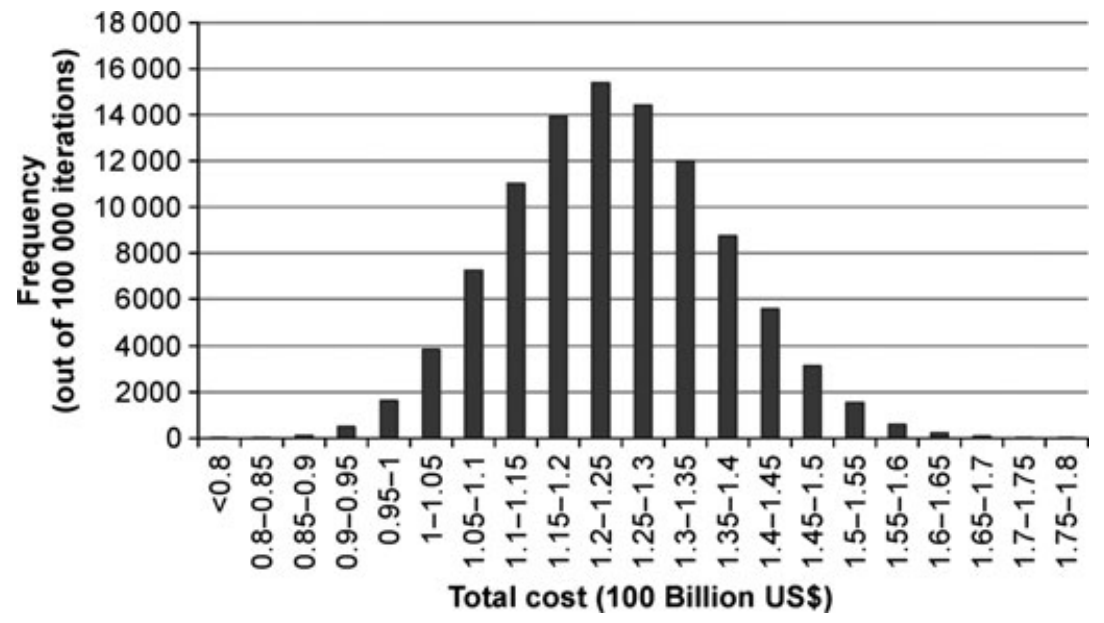

Fig. 1. Distribution of simulation results: 100000 iterations. 
Table 1. Annual primary impacts of canine rabies

\begin{tabular}{|c|c|c|c|c|}
\hline & $\begin{array}{l}\text { Latin } \\
\text { America }\end{array}$ & Africa & Asia & Total \\
\hline $\begin{array}{l}\text { Human } \\
\text { deaths }\end{array}$ & 15 & 31329 & 38090 & 69644 \\
\hline PEP cases & 624777 & 264325 & 8709365 & 9598467 \\
\hline Animal tests & 73752 & 5300 & 16500 & 95552 \\
\hline Dog vaccines & 42168802 & 6700000 & 40000000 & 88868802 \\
\hline Cattle deaths & 24 & 11500 & 21150 & 32657 \\
\hline
\end{tabular}

Table 2. Expected post-exposure prophylaxis (PEP) costs

\begin{tabular}{lccc}
\hline & Latin America & Africa & Asia \\
\hline Material cost per injection & $\$ 0.12$ & $\$ 0.12$ & $\$ 0.12$ \\
Overhead cost per visit & $\$ 0.60$ & $\$ 0.60$ & $\$ 0.60$ \\
\% patients receiving IM & 94 & 97 & 94 \\
Tissue culture vaccine unit & $\$ 11.91$ & $\$ 11.91$ & $\$ 11.91$ \\
$\quad$ cost - IM & & & \\
Visits per patient - IM & 3 & 3 & 3 \\
Injections per patient - IM & 3 & 3 & 3 \\
\% patients receiving ID & 6 & 3 & 6 \\
Tissue culture vaccine unit & $\$ 2.98$ & $\$ 2.98$ & $\$ 2.98$ \\
$\quad$ cost - ID & 3 & & \\
Visits per patient - ID & 6 & 3 & 3 \\
Injections per patient - ID & 0.175 & 0 & 6 \\
\% patients receiving hRIG & 3.325 & 1 & 0.6 \\
\% patients receiving eRIG & $\$ 29.77$ & $\$ 29.77$ & $\$ 29.77$ \\
eRIG cost per unit & $\$ 130.98$ & $\$ 130.98$ & $\$ 130.98$ \\
hRIG cost per unit & $\$ 38.03$ & $\$ 37.64$ & $\$ 39.21$ \\
Expected direct PEP cost & & & \\
per case & & & \\
\hline
\end{tabular}

Table 3. Indirect post-exposure prophylaxis (PEP) costs

\begin{tabular}{lccc}
\hline & Latin America & Africa & Asia \\
\hline Average daily income & $\$ 26.72$ & $\$ 4.77$ & $\$ 9.63$ \\
$\%$ patients accompanied by adult & 20 & 20 & 20 \\
$\%$ visitors that suffer income loss & 80 & 80 & 80 \\
Days work missed per visit & 0.5 & 0.5 & 0.5 \\
Expected income loss per visit & $\$ 10.69$ & $\$ 1.91$ & $\$ 3.85$ \\
Expected visits per PEP case & 3.6 & 3.6 & 3.6 \\
Expected income loss per PEP case & $\$ 38.48$ & $\$ 6.87$ & $\$ 13.87$ \\
Transport cost per visit & $\$ 3.45$ & $\$ 2.38$ & $\$ 4.52$ \\
Expected transport cost per PEP case & $\$ 10.36$ & $\$ 7.14$ & $\$ 13.57$ \\
\hline
\end{tabular}

Table 4. Vaccine and surveillance costs and cattle value

\begin{tabular}{llll}
\hline & Latin America & Africa & Asia \\
\hline $\begin{array}{c}\text { Dog vaccination unit } \\
\text { cost }\end{array}$ & $\$ 1.55$ & $\$ 1.55$ & $\$ 1.55$ \\
Dog control cost & - & & \\
Rabies test unit cost & $\$ 6.76$ & $\$ 1.2$ million & $\$ 30$ million \\
Cattle value (per head) & $\$ 386.99$ & $\$ 178.61$ & $\$ 6.76$ \\
\hline
\end{tabular}

Table 5. Percentage of total cost (excl. human mortality) by category and region

\begin{tabular}{lclrr}
\hline & $\begin{array}{l}\text { Latin America } \\
(\%)\end{array}$ & $\begin{array}{l}\text { Africa } \\
(\%)\end{array}$ & \multicolumn{1}{c}{$\begin{array}{l}\text { Asia } \\
(\%)\end{array}$} & \multicolumn{1}{c}{\begin{tabular}{l}
\multicolumn{1}{c}{ Sum } \\
$(\%)$
\end{tabular}} \\
\hline Direct PEP cost & 2.86 & 1.20 & 41.03 & 45.08 \\
Lost income & 2.89 & 0.22 & 14.52 & 17.62 \\
Travel cost & 0.78 & 0.23 & 14.21 & 15.21 \\
Animal testing & 0.06 & 0.00 & 0.01 & 0.08 \\
Dog vaccination & 7.84 & 1.25 & 7.44 & 16.53 \\
Dog control & 0.00 & 0.14 & 3.58 & 3.72 \\
Cattle mortality & 0.00 & 0.25 & 1.51 & 1.76 \\
Sum & 14.43 & 3.28 & 82.29 & 100.00 \\
\hline
\end{tabular}

costs, travel costs for PEP, dog control, cattle mortality and rabies diagnostic testing. While the bulk of the total cost is caused by human mortality risk, other expenses are also important. In Africa, for example, the expected expenses associated with a single PEP case are roughly equal to an entire week of the average individual's income. This represents a significant impact where the standard of living is already very low, and poverty is widespread. Additionally, some portion of the costs may be borne by governments, increasing the burden on many governments in the developing world that suffer chronic revenue shortages and already provide inadequate levels of services.

The results highlight important regional differences. The global burden falls most heavily on Asia, which accrues more than $80 \%$ of the non-human mortality costs, whereas only $3.28 \%$ of non-human mortality costs fall on Africa. While this may seem like a positive, in the light of the 31329 deaths that occur there each year, many lives could be saved if access to PEP was improved and the prevalence of canine rabies in the dog population was reduced. In fact, accounting for human mortality, $45 \%$ of the global burden falls on Africa, illustrating the disproportionate human mortality impact suffered there. Total costs in Latin America are much less than in Africa and Asia because of the low number of human deaths. This is a direct result of a coordinated effort by many countries to eliminate canine rabies (Schneider et al., 2007). The fact that Latin America appears to accrue disproportionately high non-human mortality-related costs given its low human mortality highlights an important point: vaccine and surveillance-related cost reductions from a decrease in the prevalence of the disease may take many years to materialize because the perceived risk lingers long after the actual risk has fallen.

While the mortality, PEP, dog vaccination and surveillance costs are primary impacts of canine rabies, macroeconomic impacts also arise from decreases in personal income and the resulting decrease in consumer spending. These and other macroeconomic and economic development impacts of the disease are unaccounted for in our analysis. While the consumer spending impacts of PEP and 
some other costs may be negligible because these costs represent a benefit to those who earn income from them, in the long-run, they may have other impacts. To some extent, personal expenditures on rabies-related costs may be financed from savings. Aggregate saving has an impact on the level of investment and capital accumulation that occur in an economy, which ultimately affect the pace of economic growth. Some rabies-related costs may also be borne by governments, and this causes a reallocation of public resources. Government spending on education and infrastructure are important determinants of economic development. To the extent that canine rabies forces a reallocation of resources away from these, economic growth will be stunted.

Limitations of our analysis include uncertainty surrounding parameter estimates. Some of these were derived from other studies that relied on unpublished data sources, while others are derived from complex analyses that provide a wide range of possible estimates. We illustrate how a specific level of uncertainty in each estimate affects the results using a Monte Carlo simulation, but we are unable to construct confidence intervals given that the level of uncertainty in each parameter could not be characterized. Additionally, we were unable to use a macroeconomic model specific to the study region. Unfortunately, these types of models do not exist for most developing countries. Finally, it should be pointed out that we do not consider impacts of canine rabies that occur in the Middle East, Western Asia and Eastern Europe. Lack of sufficient information on the status of the disease in these regions makes estimation of these impacts difficult. However, it is generally accepted that the majority of the impacts occur in Africa and Asia, implying this omission has little impact on the estimation of global cost. Future analyses can improve upon this analysis by including additional regions where canine rabies exists and relying on newly collected, country-specific data.

Our results provide a comprehensive assessment of the costs of the disease in three different regions of the world. Estimation of the costs is important because they are representative of the potential annual benefits that would be realized if the prevalence of the disease was substantially reduced. A complete assessment of the benefits of elimination is critical because the costs of any potential elimination effort will have to be justified. Our analysis differs from previous efforts in several important ways. We have updated a previous estimate of the cost of canine rabies in Africa and Latin America by accounting for changing PEP practices, higher incomes and population growth. Furthermore, we have estimated impacts in Latin America, a region that still incurs costs from the disease despite making considerable progress towards its elimination. The costs associated with human mortality risk have been incorporated, and these account for a large percentage of the global burden of the disease.

The global burden of canine rabies remains significant in Latin America, Africa and Asia. The majority of the global costs result from the human mortality risk attributable to the disease. Mortality in Latin America is much lower than in Africa and Asia and illustrates that successful management (and possibly elimination) of the disease can occur in developing countries. The global cost estimate is useful as an indication of the benefits that may be realized by successful management leading to a substantial reduction in the prevalence of the disease.

\section{Acknowledgements}

We thank Dr. Dennis Slate and Louise Taylor for their many helpful comments on an early draft of this manuscript.

\section{References}

Bellavance, F., G. Dionne, and M. Lebeau, 2009: The value of a statistical life: a meta-analysis with a mixed effects regression model. J. Health Econ. 28, 444-464.

Blanton, J. D., D. Palmer, and C. E. Rupprecht, 2010: Rabies surveillance in the United States during 2009. J. Am. Vet. Med. Assoc. 237, 646-657.

Bourhy, H., L. Dacheux, C. Strady, and A. Mailles, 2005: Rabies in Europe in 2005. Euro. Surveill. 10, 213-216.

Bowland, B. J., and J. C. Beghin, 2001: Robust estimates of value of statistical life for developing economics. J. Policy Model. 23, 385-396.

Doucouliagos, C., T. D. Stanley, and M. Giles, 2012: Are estimates of the value of a statistical life exaggerated? J. Health. Econ. 31, 197-206.

Global Alliance for Rabies Control 2012: International collaboration for canine rabies elimination in Latin America. Available at http://rabiescontrol.net/news/news-archive/international-collaboration-for-canine-rabies-elimination-in-latin-america.html. (accessed September 13, 2012).

Hoffman, S., P. Qin, A. Krupnick, B. Badrakh, S. Batbaatar, E. Altangerel, and L. Sereeter, 2012: The willingness to pay for mortality risk reductions in Mongolia. Resour. Energy Econ. 34, 493-513.

Knobel, D. L., S. Cleaveland, P. G. Coleman, E. M. Fevre, M. I. Meltzer, M. E. Miranda, A. Shaw, J. Zinsstag, and F. X. Meslin, 2005: Re-evaluating the burden of rabies in Africa and Asia. Bull. World Health Organ. 83, 360-368.

Lembo, T., K. Hampson, M. T. Kaare, E. Ernest, D. Knobel, R. R. Kazwala, D. T. Haydon, and S. Cleaveland, 2010: The feasibility of canine rabies elimination in Africa: dispelling doubts with data. PLoS Negl. Trop. Dis. 10, 1-9.

Miller, T. R., 2000: Variations between countries in values of statistical life. J. Transp. Econ. Policy 34, 169-188. 
Mrozek, J. R., and L. O. Taylor, 2002: What determines the value of life? A meta-analysis. J. Policy Anal. Manage. 21, 253-270.

Schneider, M. C., A. Belotto, M. P. Ade, S. Hendrickx, L. F. Leanes, M. J. Rodrigues, G. Medina, and E. Correa, 2007: Current status of human rabies transmitted by dogs in Latin America. Cad. Saude Publica. 23, 2049-2063.

Shwiff, S. A., R. T. Sterner, M. Jay-Russell, S. Parikh, A. Bellomy, M. I. Meltzer, C. E. Rupprecht, and D. Slate, 2007: Direct and indirect costs of rabies exposure: a retrospective study in Southern California (1998-2002). J. Wildl. Dis. 43, 251-257.

Shwiff, S. A., K. Hampson, and A. Anderson, 2013: Potential economic benefits of eliminating canine rabies. Antiviral Res. 98, 352-356.
Sudarshan, M. K., S. N. Madhusudana, B. J. Mahendra, D. H. Ashwath Narayana, M. S. Ananda Giri, K. Muhamuda, H. S. Ravish, and G. M. Venkatesh, 2006: Boosting effect of purified chick embryo cell rabies vaccine using intradermal route in persons previously immunized by intramuscular route or vice versa. Natl Med. J. India 19, 192-194.

Tenzin, N. K. D., and M. P. Ward, 2012: Review of rabies epidemiology and control in South, South East, and East Asia: past, present and prospects for elimination. Zoonoses Public Health 59, 451-467.

Viscusi, W. K., and J. E. Aldy, 2003: The value of a statistical life: a critical review of market estimates throughout the world. $J$. Risk Uncertainty 27, 5-76. 